\title{
Efeito Diabetogênico das Drogáas Anti-Retrovirais em Ratas Wistar Prenhes
}

\author{
Diabetogenic Effect of Antiretroviral Drugs on Pregnant Wistar Rats \\ Ernesto Antonio Figueiró-Filho, Geraldo Duarte, Patrícia El Beitune, \\ Silvana Maria Quintana, Alessandra Cristina Marcolin
}

\begin{abstract}
RESUM0
Objetivo: estudar a ação diabetogênica de drogas anti-retrovirais em ratas prenhes e o prognóstico perinatal das crias.

Métodos: estudo com ratas fêmeas prenhes adultas da raça Wistar, pesando entre 200-230 g. Foram testadas a azidotimidina (AZT), lamivudina (3TC) e nelfinavir (NFV), cujas dosagens foram padronizadas em 10 vezes a dose utilizada em gestantes, proporcionalmente ao peso dos animais. Foram avaliados sete grupos, incluindo o controle, contendo 10 ratas por grupo. O início do experimento foi o dia zero da prenhez e as cesarianas realizadas no $21^{\circ}$ dia, após decapitação, sendo os fetos contados e pesados. Procedeu-se a dosagens de glicemia, insulina, glucagon e lactato no $21^{\circ} \mathrm{dia}$. Avaliou-se também o peso do tecido adiposo retroperitoneal. Os dados foram analisados utilizando-se o teste t de Student para a análise estatística.

Resultados: os grupos tratados com 3TC, $A Z T+3 T C$ e $A Z T+3 T C+N F V$ demonstraram alterações com a redução das médias de ganho de peso materno diário, do peso da gordura retroperitoneal e peso das crias (grupo controle: 6,2 g; grupos contendo 3TC: 4,1 a 5,6 g), bem como dos valores de lactato (grupo controle: $5,8 \mathrm{mmol} / \mathrm{mL}$; grupos contendo $3 T \mathrm{C}: 3,2$ a $3,7 \mathrm{mmol} / \mathrm{mL}$ ), quando comparados ao controle. Todos os grupos tratados com drogas anti-retrovirais apresentaram redução significativa do número de fetos por ninhada (grupo controle: 14,7; grupos medicamentos: 11,1 a 12,7) e dos valores séricos de insulinemia (grupo controle: 6,2 $\mathrm{UU} / \mathrm{mL}$; grupos medicamentos: 2,1 a 2,7 $\mu \mathrm{UI} / \mathrm{mL}$ ) e elevação da glucagonemia (grupo controle: 88,2 pg/mL; grupos medicamentos: 99,7 a 120,7 pg/mL). Não houve diferenças estatisticamente significantes entre o grupo controle e tratados nos valores de glicemia.

Conclusões: o uso de anti-retrovirais em ratas prenhes causa interferência no metabolismo glicídico dos animais durante o período de prenhez, provocando significativa redução do número das crias. Observou-se que o uso do 3TC resultou em menor ganho de peso materno e das crias, redução de insulina e lactato e elevação do glucagon.
\end{abstract}

PALAVRAS-CHAVE: AIDS. Anti-retrovirais. Diabete melito. Gravidez: infecções. Prognóstico perinatal.

\section{Introdução}

A transmissão materno-fetal é a principal forma pela qual crianças são infectadas pelo vírus

Departamento de Ginecologia e Obstetrícia da Faculdade de Medicina de Ribeirão Preto da Universidade de São Paulo Correspondência:

Geraldo Duarte

Hospital das Clínicas da Faculdade de Medicina de Ribeirão Preto - USP

Avenida Bandeirantes, 2900

14049-900 - Ribeirão Preto - SP

Fone: (16) 602-2588

e-mail: gduarte@fmrp.usp.br

Trabalho realizado com apoio FAPESP (Processo 01/00084-2) da imunodeficiência humana tipo 1 (HIV-1). Sem nenhuma intervenção a transmissão vertical (TV) do HIV-1 ocorre em percentuais que variam de 14 a 35\%. Graças à utilização de anti-retrovirais profiláticos e terapêuticos, estes índices são reduzidos em mais de $70 \%{ }^{1,2}$. O primeiro ensaio clínico administrando agente anti-retroviral a gestantes para avaliação de sua segurança, tolerância e eficácia na redução da TV do HIV-1 concluiu que a administração de azidotimidina (AZT) a gestantes durante o período gestacional e durante o trabalho de parto, acrescida do uso dessa medicação ao recém-nascido, foi responsável por reduzir a TV do vírus em $67,5 \%{ }^{1}$. 
Alguns efeitos adversos maternos e fetais são conhecidos com o uso de alguns esquemas de anti-retrovirais durante a gestação. Dentre os efeitos maternos, são reconhecidos a anemia, elevação de transaminases, náuseas e vômitos, intolerância à glicose, nefrolitíase, diarréia e diabete melito $^{3,4}$. Dentre os efeitos colaterais fetais e neonatais, destacam-se a prematuridade, anemia, anasarca, anoftalmia, angioma cutâneo, criptorquidismo, costela supranumerária e hepatite transitória ${ }^{4}$.

Estudos avaliando os efeitos dos inibidores da protease em pacientes não gestantes portadores do HIV-1 demonstraram nítido aumento de triglicérides e resistência insulínica, bem como desenvolvimento de diabete melito nesses pacientes. O quadro de resistência insulínica induzida pelas drogas anti-retrovirais, geralmente cursa com hiperinsulinemia, semelhante ao diabete melito tipo 2. Em outras situações, os inibidores de proteases causam insulinopenia semelhante aos quadros de diabete melito tipo 1. No entanto, ainda não se encontram estudos aprofundados na fisiopatologia das alterações induzidas por estes fármacos ${ }^{5-7}$.

Estudos experimentais disponíveis de modelos de infecção humana pelo HIV-1 utilizam animais infectados por oncovírus indutores de leucemia ou mutados geneticamente, com a finalidade de avaliar a eficácia e efeitos terapêuticos e colaterais das drogas anti-retrovirais ${ }^{8-11}$. No entanto, em ampla revisão da literatura, constatouse a ausência de estudos sobre a ação das drogas anti-retrovirais sobre o metabolismo glicídico em animais não infectados, utilizando modelos experimentais de gestação.

Experimentalmente, pode-se induzir diabete melito em ratas prenhes e estudar as alterações funcionais maternas e fetais associadas a esse distúrbio do metabolismo de carboidratos, lípides e proteínas ${ }^{12,13}$. Outros trabalhos descrevem alterações metabólicas associadas a diabete melito induzido em ratos machos adultos ${ }^{14,15}$. Tais estudos fornecem informações importantes sobre quais são os melhores parâmetros e indicadores para serem avaliados no estudo de drogas diabetogênicas.

Atualmente existem lacunas no conhecimento do efeito das drogas anti-retrovirais sobre o metabolismo glicídico durante a gestação e, até o presente, modelos experimentais para avaliar o efeito dessas drogas sobre o metabolismo glicídico em ratas prenhes ainda não foram amplamente testados. Com base nessas informações, o objetivo do estudo proposto foi avaliar a ação diabetogênica de drogas anti-retrovirais em ratas prenhes expostas a AZT, lamivudina (3TC) e nelfinavir
(NFV), utilizando dosagens de glicemia ${ }^{12-15}$, lactato $^{16,17}$, glucagon e insulina ${ }^{14,15,18}$ e o peso da gordura retroperitoneal (peri-renal). Adicionalmente, objetivou-se a avaliação do prognóstico neonatal das crias.

\section{Métodos}

Estudo experimental realizado no Biotério do Departamento de Clínica Médica da Faculdade de Medicina de Ribeirão Preto da Universidade de São Paulo (FMRP-USP). Foram utilizadas ratas fêmeas prenhes adultas da raça Wistar, com peso inicial entre 200 e $230 \mathrm{~g}$. Os animais foram divididos aleatoriamente em sete grupos com 10 animais em cada, sendo um controle e seis grupos que receberam medicamentos anti-retrovirais isolados ou em associação. Optou-se por trabalhar com as drogas anti-retrovirais mais utilizadas durante a gestação: AZT, 3TC e NFV. Tais fármacos foram fornecidos e fracionados pela Farmácia do Hospital das Clínicas da FMRP-USP e suas dosagens padronizadas em 10 vezes a dose utilizada em pacientes gestantes, proporcionalmente ao peso dos animais. Desse modo as ratas receberam doses de $25 \mathrm{mg} /$ dia de AZT, 12,5 mg/dia de 3TC e 97,5 mg/ dia de NFV.

A via de administração dos anti-retrovirais foi orogástrica, utilizando a técnica de gavagem após diluição dos medicamentos em $5 \mathrm{~mL}$ de solução fisiológica a 0,9\%. O grupo controle recebeu solução fisiológica na mesma proporção. A administração foi sempre pela manhã, em dose única diária.

Uma vez que o período de prenhez de ratos tem duração de 21 dias, a análise dos efeitos das drogas sobre o organismo dos animais e de suas crias foi realizada no $21^{\circ}$ dia de prenhez. A experimentação teve início no "dia zero" da prenhez de todos os animais de cada grupo. A prenhez foi diagnosticada pela análise microscópica do esfregaço vaginal, sendo a observação da presença de espermatozóides no esfregaço como indicador do "dia zero" da prenhez. Os animais receberam água e alimentação ad libitum antes e durante o período de prenhez. O peso dos animais foi aferido diariamente.

No $21^{\circ}$ dia da prenhez os animais foram sacrificados por decapitação e imediatamente colhidos de 8 a $10 \mathrm{~mL}$ de sangue, proveniente da área cruenta cervical. Essa amostra sangüínea foi dividida em dois tubos, sendo o conteúdo do menor, de $1 \mathrm{~mL}$, com fluoreto, empregado para dosagem plasmática de glicemia, e o do maior, sem 
anticoagulante, para dosagens séricas de lactato, glucagon e insulina. Todo o processo de coleta sanguínea e separação de soro e plasma foi realizado sob refrigeração constante, seguido de processamento dos resultados pelo método da hexoquinase, utilizando o Cobas Mira-S da Roche para dosagem da glicemia, método automatizado, utilizando o Rapid Lab-860 da Bayer para dosagem do lactato e método do radioimunoensaio utilizando o equipamento ANSR da Abbott para dosagem do glucagon e insulina.

Após o sacrifício de cada animal, uma vez coletado o sangue, procedeu-se à realização de laparotomia para cesariana. Sucedeu-se a retirada em bloco do útero do ventre do animal e, em seguida, abertura do mesmo, possibilitando a obtenção direta dos fetos, os quais foram contados e pesados para avaliação do prognóstico neonatal. Seqüencialmente, o retroperitôneo foi explorado, dissecado, permitindo que o tecido adiposo retroperitoneal fosse removido para posterior pesagem em prazo inferior a 5 minutos pós-morte. Os dados obtidos foram submetidos à análise estatística pelo teste $t$ de Student para comparação de dois grupos. Foram considerados valores estatisticamente significativos quando o valor de $\mathrm{p}$ bicaudal foi menor que 0,05.

\section{Resultados}

Os grupos de ratas prenhes que receberam os medicamentos 3TC, AZT + 3TC e AZT + 3TC + NFV foram os que demonstraram maiores alterações ao final dos 21 dias de prenhez, com a redução das médias de ganho de peso materno diário, do peso das crias e do peso da gordura retroperitoneal. Na Tabela 1 observa-se que o grupo controle apresentou 8,6 g; 6,2 g e 3,2 g para as variáveis respectivas, ao passo que os grupos que continham 3TC apresentaram redução significativa quanto às variáveis descritas, atingindo peso materno máximo de 7,2 g e valores máximos de peso fetal de $5,6 \mathrm{~g}$ e da gordura retroperitoneal de $2,5 \mathrm{~g}$ (teste $t$ de Student, $\mathrm{p}<0,05)$.

Após 21 dias de prenhez observaram-se não apenas nos grupos com 3TC e associações, mas também em todos os grupos que receberam drogas anti-retrovirais, elevação significativa dos valores séricos de glucagon e redução dos valores séricos de insulina, bem como dos valores de lactato, quando comparados ao grupo controle. $\mathrm{O}$ grupo controle apresentou valores de $88,2 \mathrm{pg} / \mathrm{mL}$ para glucagon, $6,2 \mu \mathrm{UI} / \mathrm{mL}$ de insulina e $5,8 \mathrm{mmol} /$ $\mathrm{mL}$ de lactato, ao passo que os grupos contendo anti-retrovirais apresentaram variações compreendidas entre 2,1 e $2,7 \mu \mathrm{UI} / \mathrm{mL}$ para a insulina e 99,7 a 120,7 pg/mL para o glucagon (Tabela 2).

Tabela 1 - Ganho de peso materno diário, peso dos fetos e peso da gordura retroperitoneal com 21 dias de prenhez (média de cada grupo e desvio padrão).

\begin{tabular}{lccc}
\hline Grupo & $\begin{array}{c}\text { Peso } \\
\text { materno }(\mathrm{g})\end{array}$ & $\begin{array}{c}\text { Peso } \\
\text { fetal }(\mathrm{g})\end{array}$ & $\begin{array}{c}\text { Peso } \\
\text { gordura }(\mathrm{g})\end{array}$ \\
\hline Controle & $8,6 \pm 1,4$ & $6,2 \pm 0,6$ & $3,2 \pm 0,8$ \\
AZT & $7,8 \pm 1,5$ & $5,7 \pm 0,7$ & $3,2 \pm 0,8$ \\
3TC & $5,9 \pm 2,9^{*}$ & $4,9 \pm 1,1^{*}$ & $2,5 \pm 0,7^{*}$ \\
NFV & $8,2 \pm 1,1$ & $5,8 \pm 0,8$ & $2,5 \pm 0,7$ \\
AZT + 3TC & $7,2 \pm 1,0^{*}$ & $4,1 \pm 0,3^{*}$ & $2,0 \pm 0,6^{*}$ \\
AZT + NFV & $8,0 \pm 0,7$ & $6,0 \pm 0,2$ & $3,1 \pm 0,8$ \\
AZT + 3TC + NFV & $6,9 \pm 1,0^{*}$ & $5,6 \pm 0,4^{*}$ & $2,2 \pm 0,9^{*}$ \\
\hline
\end{tabular}

${ }^{*} p<0,05$. Controle vs medicamentos. Teste $t$ de Student.

Grupos com $n=10$ animais.

Tabela 2 - Valores de lactato, insulina e glucagon materno no $21^{\circ}$ dia de prenhez (média de cada grupo e desvio padrão).

\begin{tabular}{lccc} 
Grupo & $\begin{array}{c}\text { Lactato } \\
(\mathrm{mmol} / \mathrm{mL})\end{array}$ & $\begin{array}{c}\text { Insulina } \\
(\mu \mathrm{UI} / \mathrm{mL})\end{array}$ & $\begin{array}{c}\text { Glucagon } \\
(\mathbf{p g} / \mathrm{mL})\end{array}$ \\
\hline Controle & $5,8 \pm 1,9$ & $6,2 \pm 0,9$ & $88,2 \pm 5,5$ \\
AZT & $5,9 \pm 1,9$ & $2,7 \pm 1,4^{*}$ & $109,2 \pm 22,7^{*}$ \\
3TC & $3,7 \pm 1,3^{*}$ & $2,3 \pm 1,8^{*}$ & $113,6 \pm 36,1^{*}$ \\
NFV & $5,3 \pm 2,0$ & $2,2 \pm 1,5^{*}$ & $114,4 \pm 31,6^{*}$ \\
AZT + 3TC & $3,6 \pm 0,8^{*}$ & $2,1 \pm 1,5^{*}$ & $116,2 \pm 21,9^{*}$ \\
AZT + NFV & $4,6 \pm 1,5$ & $2,1 \pm 1,4^{*}$ & $99,7 \pm 10,5^{*}$ \\
AZT + 3TC + NFV & $3,2 \pm 1,1^{*}$ & $2,1 \pm 1,1^{*}$ & $120,7 \pm 37,3^{*}$
\end{tabular}

${ }^{*} \mathrm{p}<0,05$. Controle vs medicamentos. Teste $t$ de Student Grupos com $n=10$ animais.

Na Tabela 3 observa-se média de 14,7 fetos por ninhada para o grupo controle, ao passo que os grupos que utilizaram anti-retrovirais apresentaram valores que não superaram 12,7 fetos por ninhada (teste $t$ de Student, $\mathrm{p}<0,05$ ).

Tabela 3 - Numero de fetos por animal no $21^{\circ}$ dia de prenhez (média de cada grupo e desvio padrão).

\begin{tabular}{lc}
\hline Grupo & Número de fetos \\
\hline Controle & $14,7 \pm 1,4$ \\
AZT & $12,1 \pm 2,3^{\star}$ \\
$3 T C$ & $11,1 \pm 4,3^{\star}$ \\
NFV & $12,7 \pm 2,2^{*}$ \\
AZT + 3TC & $11,5 \pm 2,4^{*}$ \\
AZT + NFV & $11,5 \pm 2,4^{*}$ \\
AZT + 3TC + NFV & $11,4 \pm 3,8^{\star}$
\end{tabular}

${ }^{*} p<0,05$. Controle vs medicamentos. Teste $t$ de Student. Grupos com $n=10$ animais. 
Por outro lado, não houve diferenças significantes entre o grupo controle e todos os grupos tratados com medicamentos anti-retrovirais quanto às dosagens de glicemia (controle: $87,5 \mathrm{mg}$ / dL; tratados: 83,7 a 95,5 mg/dL).

\section{Discussão}

No presente estudo avaliaram-se o efeito dos medicamentos anti-retrovirais sobre o metabolismo glicídico de ratas prenhes e seus efeitos sobre o prognóstico neonatal das crias. Utilizaram-se os parâmetros de dosagens mais comumente citados para a avaliação do efeito diabetogênico dos anti-retrovirais no organismo dos animais, como dosagens séricas de glicose, insulina, glucagon e lactato, bem como avaliação do ganho de peso materno e peso da gordura retroperitoneal. No intuito de avaliar o prognóstico neonatal, avaliaramse o número e peso dos fetos.

Nos trabalhos pesquisados é relatado aumento significativo dos niveis glicêmicos nos animais que desenvolvem diabete melito experimentalmente $\mathrm{e}^{12-15}$. Ocorre, também, aumento dos níveis plasmáticos de lactato em ratas prenhes diabéticas, bem como nas suas crias, fato indicativo da ação diabetogênica das drogas utilizadas ${ }^{16,17}$. No presente estudo não foram observadas alterações significativas nos níveis de glicemia dos animais que receberam medicamentos anti-retrovirais em relação aos do grupo controle. Esses achados são similares aos observados em estudo clínico realizado nesse departamento, onde aferiram-se as curvas glicêmicas em gestantes portadoras do HIV-1, usuárias de anti-retrovirais. No estudo em questão não se identificaram diferenças significativas em relação ao grupo controle, em coletas obtidas até a $32^{\mathrm{a}}$ semana de gestação, observando-se elevação significativa, porém limítrofe, no período compreendido entre 33 e 38 semanas de gestação no grupo em uso de anti-retrovirais combinados, em relação ao grupo controle ${ }^{19}$. Esses resultados indicam lesão parcial no pâncreas materno causada pelos anti-retrovirais, vindo possivelmente a traduzir-se como diabete clínico.

Todavia, em todos os grupos de animais tratados com drogas anti-retrovirais observara-se redução dos níveis de insulina e elevação dos valores de glucagon no $21^{\circ}$ dia de prenhez. As dosagens de insulina e glucagon são auxiliares no acompanhamento do efeito diabetogênico de medicamentos no organismo de ratos, ocorrendo redução dos niveis insulinêmicos e elevação dos níveis do glucagon nos casos de lesão pancreática e desenvolvimento de diabete melito tipo $1^{14,15}$. Nos casos de resistência à insulina, pode ocorrer aumento dos seus níveis, o que sugere um quadro de diabete melito tipo $2^{18}$. O aumento dos níveis plasmáticos de glucagon e a redução da insulina estimulam a gliconeogênese, favorecendo também a degradação do glicogênio hepático.

Apesar dos valores normais de glicemia verificados nos animais do presente estudo, as alterações descritas de insulina e glucagon comprovam efeito metabólico direto sobre o pâncreas materno ${ }^{20}$. A insulinopenia como marcador de lesão pancreática já foi bem estabelecida por Eizirik e Migliorini ${ }^{14}$ e Eizirik et al. ${ }^{15,21}$ em seus estudos com ratos diabéticos. Outros autores, também comprovaram efeito diabetogênico experimental em ratos $^{22}$ e tartarugas ${ }^{23}$ por meio da marcação imuno-histoquímica do parênquima pancreático, que revelou redução significativa de células beta produtoras de insulina, confirmando a relação de insulinopenia e efeito medicamentoso lesivo sobre o pâncreas.

Dentre todos os medicamentos testados, os grupos submetidos ao tratamento com 3TC e suas associações foram os que demonstraram alterações significativas em relação ao grupo controle, tanto do ponto de vista metabólico materno quanto do ponto de vista do prognóstico fetal. Houve, nesses grupos, menor ganho de peso materno, menor peso fetal e menor número de fetos por ninhada em comparação ao grupo controle.

A redução do peso da gordura retroperitoneal é alteração reconhecida como indicador do efeito diabetogênico em ratos ${ }^{14,15,21}$. Apesar dos níveis glicêmicos normais dos animais, os resultados da presente casuística demonstram redução do peso da gordura retroperitoneal, redução da insulina e elevação do glucagon. Esses achados podem ser indicativos do efeito diabetogênico ainda inicial, secundário ao uso dos anti-retrovirais durante o limitado período da prenhez.

O menor ganho do peso materno pode ter sido conseqüência do menor ganho do peso das crias, e pode adicionalmente estar associado à redução da gordura retroperitoneal. Em trabalho recente, com metodologia semelhante, Figueiró-Filho et al. ${ }^{24}$ demonstraram as mesmas alterações de peso materno e das crias. Possivelmente, a redução do peso da gordura retroperitoneal e o menor ganho de peso materno possam estar também associados a ações das drogas anti-retrovirais em outros aspectos não conclusivamente elucidados.

Ao contrário do que é descrito por outros autores $^{16,17}$, os quais descrevem elevação dos valores de lactato em ratos diabéticos e em suas crias, esses achados não foram confirmados na 
presente casuística, possivelmente por não se identificar diabete clínico no período de 21 dias de exposição aos anti-retrovirais.

Quanto aos aspectos perinatais das crias, o presente estudo demonstrou redução do peso fetal nos grupos cujo tratamento continha o 3TC e redução do número dos fetos por ninhada em todos os grupos, inclusive nos que receberam anti-retrovirais isoladamente. Esses dados também corroboram os achados de Figueiró-Filho et al. ${ }^{24}$, que demonstraram efeitos importantes dos esquemas de anti-retrovirais que continham o 3TC sobre as taxas de fertilidade em ratas prenhes, principalmente no aumento das perdas fetais pós-implantação e redução da viabilidade fetal dos animais expostos a esses medicamentos.

Os resultados advindos do presente estudo oferecem apoio à afirmação proposta por Riecke et al. ${ }^{25}$, que sugerem a realização de estudos para se avaliar a toxicidade e efeitos adversos, notadamente o efeito diabetogênico de medicamentos anti-retrovirais isolados e em associação, de forma a determinar a segurança do uso desses fármacos no período gestacional.

\section{ABSTRACT}

Purpose: to experimentally evaluate the diabetogenic effects of antiretroviral drugs on pregnant Wistar rats and the perinatal effects on the offspring.

Methods: adult female pregnant Wistar rats weighing 200$230 \mathrm{~g}$ were used. The antiretroviral drugs zidovudine (ZDV), lamivudine (3TC) and nelfinavir (NFV) were used alone and in association at daily doses of ten times the dose normally used in pregnant women, proportionally to the animal's body weight. Seven groups were studied, including the control. The experiment started on day 0 of pregnancy and the pregnant animals were sacrificed on day 21. The fetuses were counted and weighed. Blood determinations of glucose, insulin, glucagon and lactate were performed on day 21. The retroperitoneal adipose tissue was weighed. Data were analyzed statistically by Student's t-test.

Results: the groups treated with $3 T C, Z D V+3 T C$ and $Z D V+$ $3 T C+N F V$ showed decreasing values of maternal daily body weight gain, retroperitoneal adipose tissue weight and weight of fetuses (control group: $6.2 \mathrm{~g}$; $3 T C$ group variation: 4.1-5.6 g). The serum lactate levels were also decreased when compared to the control in these groups (control group: 5.8 $\mathrm{mmol} / \mathrm{mL}$; $3 T C$ group variations: 3.2-3.7 $\mathrm{mmol} / \mathrm{mL}$ ). All antiretroviral-treated groups showed a decreasing number of fetuses when compared to the control (control group: 14.7; drug group variation: 11.1-12.7). All treated groups also showed decreasing serum values of insulin (control group: $6.2 \mu \mathrm{IU} / \mathrm{mL}$; drug group variation: 2.1 to $2.7 \mu \mathrm{IU} / \mathrm{mL}$ ) and increasing serum levels of glucagon when compared to the control (control group: $88.2 \mathrm{pg} / \mathrm{mL}$; drug group variation:
99.7 to $120.7 \mathrm{pg} / \mathrm{mL}$ ). There was no statistical significance of glucose levels when comparing treated groups to the control. Conclusions: the antiretroviral drugs interfered in carbohydrate metabolism of pregnant rats and reduced the number of fetuses. 3TC caused less maternal body weight gain, decreased fetus weight and lactate and insulin levels and increased serum glucagon.

KEYWORDS: Antiretroviral drugs. Diabetes mellitus. HIV and pregnancy. Pregnancy outcome.

\section{Referências}

1. Connor EM, Sperling RS, Gelber R, et al. Reduction of maternal-infant transmission of human immunodeficiency virus type 1 with zidovudine treatment. N Engl J Med 1994; 331:1173-80.

2. Lindegren ML, Byers RH Jr, Thomas P, et al. Trends in perinatal transmission of HIV/AIDS in the United States. JAMA 1999; 282:531-8.

3. Martin R, Boyer P, Hammill H, et al. Incidence of premature birth and neonatal respiratory disease in infants of HIV-positive mothers. J Pediatr 1997; 131:851-6.

4. Lorenzi P, Spicher VM, Laubereau B, et al. Antiretroviral therapies in pregnancy: maternal, fetal and neonatal effects. AIDS 1998; 12:F241-7.

5. Dube MP, Johnson DL, Currier JS, Leedom JM. Protease inhibitor-associated hyperglycaemia. Lancet 1997; 350:713-4.

6. Visnegarwala F, Krause KL, Musher DM. Severe diabetes associated with protease inhibitor therapy [Letter]. Ann Intern Med 1997; 127:947.

7. Yanovski JA, Miller KD, Kino T, et al. Endocrine and metabolic evaluation of human immunodeficiency virus-infected patients with evidence of protease inhibitor-associated lipodystrophy. J Clin Endocrinol Metab 1999; 84:1925-31.

8. Falcone A, Darnowski JW, Ruprecht RM, Chu SH, Brunetti I, Calabresi P. Different effect of benzylacylouridine on the toxic and therapeutic effects of azidothymidine in mice. Blood 1990; 76:2216-21.

9. Ohnota H, Okata Y, Ushijima H, Kitamura T, Komura K, Mizuochi T. 3'-Azido-3'-deoxythymidine prevents induction of murine acquired immunodeficiency syndrome in C57BL/10 mice infected with LP-BM5 murine leukemia viruses, a possible animal model for antiretroviral drug screening. Antimicrob Agents Chemother 1990; 34:605-9.

10.Ruprecht RM, Bernard LD, Gama Sosa MA, et al. Murine models for evaluating antiretroviral therapy. Cancer Res 1990; 50:5618S-5627S. 
11.Toltzis P, Marx CM, Kleinman N, Levine EM, Schmidt EV. Zidovudine-associated embryonic toxicity in mice. J Infect Dis 1991; 163:1212-8.

12.Rudge MV, Calderon IM, Ramos MD, Rodrigues MA. Diabetes and experimental pregnancy in rats: course of maternal blood glucose levels and its repercussions on the blood glucose levels and pancreas of newborn pups. Braz J Med Biol Res 1995; 28:219-25.

13. Rudge MVC, Gomes CMM, Calderon IMP, et al. Study of the evolution of the placenta and fetal pancreas in the pathophysiology of growth retardation intrauterine due to restricted maternal diet. São Paulo Med J 1999; 117:49-56.

14.Eizirik DL, Migliorini RH. Reduced diabetogenic effect of streptozotocin in rats previously adapted to a high-protein, carbohydrate-free diet. Diabetes 1984; 33:383-8.

15.Eizirik DL, Tze WJ, Tai J, Migliorini RH. Effects of a high protein diet on the evolution of diabetes in streptozotocin-induced and spontaneously diabetic “BB” Wistar rats. Acta Diabetol Lat 1986; 23;107-16.

16.Jawerbaum A, Gonzalez ET, Catafau JR, et al. Glucose, glycogen and triglyceride metabolism, as well as prostaglandin production in uterine strips and in embryos from diabetic pregnant rats. Influences of the presence of substrate in the incubation medium. Prostaglandins 1993; 46:417-31.

17. Chitra CL, Cuezva JM, Patel MS. Changes in the activity of "active" pyruvate dehydrogenase complex in the newborn of normal and diabetic rats. Diabetologia 1985; 28:148-52.
18.Kahn CR. Diabetes. Causes of insulin resistance. Nature 1995; 373:384-5.

19.El Beitune P, Duarte G, Quintana SM, et al. Intolerância glicêmica e o prognóstico perinatal em gestantes utilizando anti-retrovirais. Rev Bras Ginecol Obstet 2003; 25:465-71.

20.Kettelhut IC, Foss MC, Migliorini RH. Glucose homeostasis in a carnivorous animal (cat) and in rats fed a high-protein diet. Am J Physiol 1980; 239:R437-44.

21.Eizirik DL, Boschero AC, Migliorini RH. Previous adaptation to a high-protein diet protects against streptozotocin-induced inhibition of insulin release from isolated rats islets. Braz J Med Biol Res 1985; 18:233-5.

22.Miyahara T, Kawabuchi M, Goto M, Nakano I, Nada O, Nawata H. Morphological study of pancreatic endocrine in an experimental chronic pancreatitis with diabetes induced by stress and cerulein. Ultrastruct Pathol 1999; 23:171-80.

23. Muniz WW, Marques M. Glycemia and immunohistochemical changes in the endocrine pancreas of the turtle Chrysemys dorbigni treated with streptozotocin. Braz J Med Biol Res 1989; 22:1033-7.

24.Figueiró-Filho EA, Duarte G, Rosa e Silva AAM, et al. Efeito das drogas anti-retrovirais sobre as taxas de fertilidade de ratas Wistar. Rev Bras Ginecol Obstet 2002; 24:647-52.

25.Riecke K, Schulz TG, Shakibaei M, Krause B, Chahoud I, Stahlmann R. Developmental toxicity of the HIV-protease inhibitor indinavir in rats. Teratology 2000; 62:291-300.

Recebido em: 31/10/2003 Aceito com modificações em: 7/1/2004 